\title{
Review of: "Biologically synthesized iron nanoparticles (FeNPs) from Phoenix dactylifera have anti-bacterial activities"
}

\author{
Suvardhan Kanchi
}

Potential competing interests: The author(s) declared that no potential competing interests exist.

This study deals with the green synthesis of iron nanoparticles (FeNPs) using Phoenix dactylifera extract. The as-synthesized NPs were characterized with different techniques including UV-Visible spectroscopy, Fourier transform infrared spectroscopy (FTIR), and nano zeta-sizer analysis. The obtained NPs from this study was successfully applied to evaluate the antibacterial capabilities against various microbes such as Escherichia coli, Bacillus subtilis, Micrococcus leutus, and Klebsiella pneumoniae.

There are several concerns involved in this study, listed below:

1. There is no justification for the word "Biologically" in the title to that of the content in the paper.

2. It is not sure to authors whether the NPs synthesized in this paper are zero valent metallic iron (ZVMI) and iron oxide $\left(\mathrm{Fe}_{2} \mathrm{O}_{3} / \mathrm{Fe}_{3} \mathrm{O}_{4}\right)$.

3. Authors has claimed that sharp peaks were observed at $450 \mathrm{~nm}$, which is not true as seen from Figure 2. Two peaks were observed between $300-350 \mathrm{~nm}$ and $500-550 \mathrm{~nm}$. However, in my opinion, the UVvis spectrum in the paper is not original or instrumental generated. Authors have drew it with excel, which is not acceptable.

4. In Figure 3, FTIR shows only the leaf extract of Phoenix dactylifera. It is very important to include FTIR spectrum for NPs, to distinguish between extract and NPs. These results could lead to deduce the role and their responsibilities of biomolecules present in the extract. On the other hand, the Figure 3 FTIR spectrum was an scanned image and originality is questionable.

5. In Figure 4, the DLS shows the average size of the particles in between $950-3000 \mathrm{~nm}$ as per authors statement. In general, particles above $1000 \mathrm{~nm}$ are not NPs, but throughout the study authors has claimed as NPs. The results from Figure 1 are in contradictory with Figure 4.

6. It is wondered that the Discussion section of this paper looks like a LITERATURE REVIEW. There is nothing constructive discussion found on the authors results.

7. In this study, authors has focused on the characterization techniques such as UV-vis, DLS and FTIR, but ignored very important techniques like TEM/HR-TEM/SEM, XRD etc. With these two techniques author would have been confirmed the actual size of the NPs as well as to support Figure 4, but it was not the case in the paper.

8. I agree with the authors that Phoenix dactylifera is of highly significant tree, however there is no either GC/LC-MS studies to confirm the phytochemicals present in the extract of leaves. This would have 
supported the FTIR results.

9. The in-depth discussion on the antibacterial activity of FeNPs was missing in the paper. The size of the NPs is crucial in the microbial studies due to their interaction with microbial cell membranes. In general, the effectiveness of the microbial activity decreases with large sized particles. Unfortunately, the discussion on the antibacterial activity is insufficient and insignificant. 